\title{
Adaptive-measurement based quantum tomography
}

\author{
Shao-Ming Fei* \\ School of Mathematical Sciences, Capital Normal University, Beijing 100048, China
}

Received October 24, 2016; accepted October 25, 2016; published online November 21, 2016

Citation: $\quad$ S. M. Fei, Adaptive-measurement based quantum tomography, Sci. China-Phys. Mech. Astron. 60, 020331 (2017), doi: 10.1007/s11433-016-0416-3

Quantum state tomography (QST) concerns a complete description of arbitrary unknown quantum states by using experimental data from quantum measurements. The adaptive measurement, i.e., repeated measurement of an observable utilizing prior measurement outcomes and adapting to changing experimental conditions, has great potential for various purposes in quantum information sciences like QST. An open problem left for QST is how many measurements are needed to determine unknown quantum states.

In fact, the problem of how many measurements are needed to determine a wave function of a quantum system is quite nontrivial, and has attracted considerable attention over the history of the subject [1-3]. The development of quantum information science has shed new light on this problem, which is just rephrased by QST for pure states in the language of quantum information. In particular, the precise meaning of the word 'determine' is clarified, where two important scenarios are considered. One is UDP (i.e. no other pure states can give the same measurement result) and UDA (i.e. no other states, pure or mixed, can give the same measurement result) [4]. Another one is whether the measurement results determine all pure states (i.e. any state can be reconstructed unambiguously) or just generic pure states.

The problem of QST for pure states is naturally extended to the problem of determining unknown quantum processes, i.e., quantum process tomography (QPT). For unitary channels QPT is aimed to determine an unknown unitary operation by using experimental data. Mathematically, QPT may be reduced to QST in a larger Hilbert space.

By using adaptive measurements the QST for pure states

\footnotetext{
*Corresponding author (email: feishm@cnu.edu.cn)
}

and QPT for unitary channels are studied in ref. [5]. For QST, it is shown that $2 d-1$ measurements are enough to UDA (hence UDP) for all pure states by using adaptive measurements, which significantly improves the $4 d-5$ lower bound for UDP by using non-adaptive measurements [6]. Furthermore, by applying the protocol to study QPT, the authors in ref. [5] show that $d^{2}+d-1$ measurements are sufficient to re-construct all unitary channels when adaptive scheme is allowed. The summarized results are shown in Table 1 from ref. [5].

The nuclear magnetic resonance system has been used for experimental demonstration for many quantum information processing, e.g. refs. [11-13]. To implement experimentally the adaptive unitary process tomography (AUPT) protocol in a 2-qubit nuclear magnetic resonance system and examine the performance of the AUPT protocol, in ref. [5] five elementary gates $H_{1}=H \otimes I, H_{2}=I \otimes H, T_{1}=T \otimes I, T_{2}=I \otimes T$ and $C N O T_{12}$ are used, accounting to the fact that any 2-qubit quantum circuit can be decomposed into these five gates in arbitrary accuracy [14], where $H$ is the Hadamard gate, $T$ is the $\pi / 8$ phase gate, and $C N O T_{12}$ is the controlled-NOT gate. Starting from the thermal equilibrium state, four pseudo-pure states $|00\rangle,|01\rangle,|10\rangle$ and $|11\rangle$ are created [15]. Due to the unitary channel, the output quantum states are superposition

Table 1 Comparison of the best known number of measurements needed for different methods [5]

\begin{tabular}{cccc}
\hline & UDP & UDA & UPT \\
\hline All (nonadaptive) & $4 d-5[6]$ & $5 d-7[4]$ & $4 d^{2}-2 d-4[7]$ \\
Generic (nonadaptive) & $2 d-1[8]$ & $2 d-1[9]$ & $d^{2}+d-1[10]$ \\
All (adaptive) & $2 d-1$ & $2 d-1$ & $d^{2}+d-1$ \\
\hline
\end{tabular}


Table 2 Average fidelities of the standard QPT and the AUPT protocol compared to the theoretical gate, respectively [5]

\begin{tabular}{cccccc}
\hline Average fidelity & $H_{1}$ & $H_{2}$ & $T_{1}$ & $T_{2}$ & $C N O T_{12}$ \\
\hline QPT & $0.9903 \pm 0.0005$ & $0.9850 \pm 0.0008$ & $0.9855 \pm 0.0007$ & $0.9937 \pm 0.0003$ & $0.9861 \pm 0.0006$ \\
AUPT & $0.9826 \pm 0.0010$ & $0.9863 \pm 0.0008$ & $0.9619 \pm 0.0023$ & $0.9495 \pm 0.0018$ & $0.9350 \pm 0.0033$ \\
\hline
\end{tabular}

states. By measuring the module and relative phase of each coefficients separately, the unitary process can be characterized. In total, the numbers of experiments needed are 42 . As a comparison, a standard QPT for each gate needs 240 experiments. The experimental results [5] in Table 2 show that, without considerable loss of fidelity, the AUPT protocol that reconstructs unitary channels via adaptive measurements significantly reduces the number of experiments required by the standard QPT.

1 W. Pauli, Die allgemeinen Prinzipien der Wellenmechanik (Springer, Berlin, Heidelberg, 1933).

2 S. Weigert, Phys. Rev. A 45, 7688 (1992).

3 J. P. Amiet, and S. Weigert, J. Phys. A-Math. Gen. 32, 2777 (1999).

4 J. Chen, H. Dawkins, Z. Ji, N. Johnston, D. Kribs, F. Shultz, and B. Zeng, Phys. Rev. A 88, 012109 (2013).

5 H. Y. Wang, W. Q. Zheng, N. K. Yu, K. R. Li, D. W. Lu, T. Xin, C. Li,
Z. F. Ji, D. Kribs, B. Zeng, X. H. Peng, and J. F. Du, Sci. China-Phys. Mech. Astron. 59, 100313 (2016).

6 T. Heinosaari, L. Mazzarella, and M. M. Wolf, Commun. Math. Phys. 318, 355 (2013).

7 G. Gutoski, and N. Johnston, J. Math. Phys. 55, 032201 (2014).

8 J. Finkelstein, Phys. Rev. A 70, 052107 (2004).

9 C. H. Baldwin, I. H. Deutsch, and A. Kalev, Phys. Rev. A 93, 052105 (2016).

10 C. H. Baldwin, A. Kalev, and I. H. Deutsch, Phys. Rev. A 90, 012110 (2014).

11 Y. Long, G. R. Feng, J. Pearson, and G. L. Long, Sci. China-Phys. Mech. Astron. 57, 1256 (2014).

12 F. Z. Jin, H. W. Chen, X. Rong, H. Zhou, M. J. Shi, Q. Zhang, C. Y. Ju, Y. F. Cai, S. L. Luo, X. H. Peng, and J. F. Du, Sci. China-Phys. Mech. Astron. 59, 630302 (2016).

13 X. L. Zhen, T. Xin, F. H. Zhang, and G. L. Long, Sci. China-Phys. Mech. Astron. 59, 690312 (2016).

14 M. A. Nielsen, I, L. Chuang, Quantum Computation and Quantum Information (Cambridge University Press, Cambridge, 2010).

15 D. G. Cory, A. F. Fahmy, and T. F. Havel, Proc. Natl. Acad. Sci. 94, 1634 (1997). 